\title{
Nucleus Accumbens-Derived Glial Cell Line-Derived Neurotrophic Factor Is a Retrograde Enhancer of Dopaminergic Tone in the Mesocorticolimbic System
}

\author{
Jun Wang, ${ }^{1}$ Sebastien Carnicella, ${ }^{1,3}$ Somayeh Ahmadiantehrani, ${ }^{1,2}$ Dao-Yao He, ${ }^{1}$ Segev Barak, ${ }^{1}$ Viktor Kharazia, ${ }^{1}$ \\ Sami Ben Hamida, ${ }^{1}$ Agustin Zapata, ${ }^{4}$ Toni S. Shippenberg, ${ }^{4}$ and Dorit Ron ${ }^{1,2,3}$ \\ ${ }^{1}$ Gallo Research Center, Emeryville, California 94608, ${ }^{2}$ Graduate Program in Pharmaceutical Sciences and Pharmacogenomics and ${ }^{3}$ Department of \\ Neurology, University of California, San Francisco, California 94143, and ${ }^{4}$ Integrative Neuroscience Section, National Institute on Drug Abuse, Baltimore, \\ Maryland 21224
}

Spontaneous firing of ventral tegmental area (VTA) dopamine (DA) neurons provides ambient levels of DA in target areas such as the nucleus accumbens $(\mathrm{NAc}$ ) and the prefrontal cortex (PFC). Here we report that the glial cell line-derived neurotrophic factor (GDNF), produced in one target region, the NAc, is retrogradely transported by DA neurons to the VTA where the growth factor positively regulates the spontaneous firing activity of both NAc- and PFC-projecting DA neurons in a mechanism that requires the activation of the mitogenactivated protein kinase (MAPK) pathway. We also show that the consequence of GDNF-mediated activation of the MAPK signaling cascade in the VTA is an increase in DA overflow in the NAc. Together, these results demonstrate that NAc-produced GDNF serves as a retrograde enhancer that upregulates the activity of the mesocorticolimbic DA system.

\section{Introduction}

Glial cell line-derived neurotrophic factor (GDNF) was originally identified as a potent survival factor for midbrain dopamine (DA) neurons in culture (Lin et al., 1993). The growth factor was subsequently shown to be critical for the development, survival, maintenance, and function of a number of other populations of neurons in the CNS, such as hippocampal neurons (Ledda et al., 2007), motor neurons, as well as sympathetic and sensory neurons in the peripheral nervous system (Henderson et al., 1994).

GDNF is essential for postnatal development (Granholm et al., 2000) and maintenance of midbrain DA neurons in the adult brain (Pascual et al., 2008) and for the protection of midbrain DA neurons from neurotoxic damage (Tomac et al., 1995a). Because of these properties, the growth factor has been considered as a

Received July 27, 2010; revised Aug. 24, 2010; accepted Aug. 31, 2010.

This work was supported by National Institutes of Health ( $\mathrm{NIH}$ )-National Institute on Alcohol Abuse and Alcoholism (NIAAA) Grant R01 AA014366 (D.R.); the State of California for Medical Research on Alcohol and Substance Abuse through the University of California, San Francisco (D.R.); the NIH-National Institute on Drug Abuse Intramural Research Program (T.S.S.); National Research Service Award-NIAAA Grant F31 AA017801 (S.A.); the Merck Scholar Award (S.A.); and Department of the Army Grant W81XWH-07-1-0079 (D.R.), for which the U.S. Army Medical Research Acquisition Activity (Fort Detrick, MD) is the awarding and administering acquisition office. The content of the information represented does not necessarily reflect the position or the policy of the Government, and no official endorsement should be inferred. We thank Wenheng Zhu for technical support; Dr. Elyssa Margolis for technical support, helpful discussions, and critical review of this manuscript; Drs. Billy Chen, Robert Froemke, and Patricia Janak for critical review of this manuscript; and Drs. Howard Fields and Greg Hjelmstad for helpful discussions.

Correspondence should be addressed to Dr. Dorit Ron, 5858 Horton Street, Suite 200, Emeryville, CA 94608. E-mail:dron@gallo.ucsf.edu.

S. Carnicella's present address: Grenoble Institute of Neuroscience, Inserm U836, F-38706 Grenoble, France. DOI:10.1523/JNEUROSCI.3909-10.2010

Copyright $\odot 2010$ the authors $\quad 0270-6474 / 10 / 3014502-11 \$ 15.00 / 0$ potential treatment for Parkinson's disease, which is characterized by the loss of DA neurons in the substantia nigra (SN) (Deierborg et al., 2008). In addition, accumulating evidence suggests that GDNF in the mesolimbic system, consisting of DAergic projections from the midbrain ventral tegmental area (VTA) to the nucleus accumbens (NAc), plays a role in addiction (Carnicella and Ron, 2009; Lu et al., 2009).

GDNF $\mathrm{mRNA}$ is produced in the striatum (the primary target area of midbrain DA neurons), whereas the basal level of the growth factor in the midbrain is very low (Pochon et al., 1997; Trupp et al., 1997). However, the GDNF receptor complex, consisting of the GDNF family receptor $\alpha 1$ (GFR $\alpha 1$ ) and the receptor tyrosine kinase Ret, is highly expressed in the midbrain but not in the striatum (Trupp et al., 1997; Sarabi et al., 2001; Jain et al., 2006). In line with these observations, Tomac et al. (1995b) reported that GDNF is retrogradely transported by DA neurons to the SN. In the DAergic neurons of the midbrain, ligation of GDNF with GFR $\alpha 1$ leads to the recruitment and activation of Ret and to the consequent activation of several signaling cascades including the mitogen-activated protein kinase (MAPK) and phosphoinositide 3 kinase (PI3K) pathways (Airaksinen and Saarma, 2002).

Yang et al. (2001) previously reported that acute ex vivo treatment of midbrain neurons with recombinant GDNF increases the evoked firing rate of DA neurons. Within the striatum, GDNF mRNA levels are higher in the NAc (the target region of VTA DA neurons) than in the dorsal striatum (the target region of SN DA neurons) (Barroso-Chinea et al., 2005). Therefore, the aim of the present study was to determine whether endogenous GDNF in the NAc is a retrograde factor that regulates the firing activity of DA neurons in the VTA. 


\section{Materials and Methods}

Animals

Unless otherwise indicated, male Sprague Dawley rats (21-29 d old) were used. Rats were housed under a $12 \mathrm{~h}$ light/dark cycle, with lights on at 7:00 A.M., and food and water were available ad libitum. All animal procedures in this study were approved by the Gallo Research Center Institutional Animal Care and Use Committee and were conducted in agreement with the National Research Council Guide for the Care and Use of Laboratory Animals (1996).

\section{Reagents}

Recombinant human GDNF was obtained from R\&D Systems. Tetrodotoxin (TTX), 2,3-dioxo-6-nitro-1,2,3,4-tetrahydrobenzo[f] quinoxaline7-sulfonoamide (NBQX), MK-801, PD 98059, and LY 294002 were purchased from Tocris. Neuro-DiI was purchased from Biotium. Picrotoxin, $\mathrm{R}(+)$ Baclofen, 6-hydroxydopamine (6-OHDA), desipramine, and all ingredients of the intracellular and external solutions were obtained from Sigma. The polyclonal phospho-extracellular signalregulated kinase 1/2 (p-ERK1/2) antibody used for immunohistochemistry was purchased from Cell Signaling Technology. The monoclonal anti-tyrosine hydroxylase (TH) antibody used for immunohistochemistry was purchased from Sigma, and the polyclonal anti-TH antibody used for postrecording staining was obtained from Millipore Bioscience Research Reagents. Horseradish peroxidase-conjugated secondary antibodies were purchased from Santa Cruz Biotechnology. The secondary antibodies Alexa Fluor 488-labeled donkey anti-rabbit and Alexa Fluor 594-labeled donkey anti-mouse were purchased from Invitrogen. Cy5 anti-rabbit secondary antibody, FITC goat anti-rabbit IgG, and fluorescein [5-(4,6-dichlorotriazinyl)aminofluorescein] streptavidin were obtained from Jackson ImmunoResearch. The TRIzol reagent was purchased from Invitrogen. The oligo $(\mathrm{dT})$ primers and the reverse transcription system used for reverse transcription of mRNA into cDNA were obtained from Promega. Phosphatase inhibitor cocktails I and II were obtained from Sigma. The polyclonal antibodies, anti-Ret, and antiphospho-Ret (anti-pRet, Tyr1062) and the monoclonal anti-pERK1/2 antibodies were purchased from Santa Cruz Biotechnology. Protease inhibitor mixture was purchased from Roche. The BCA Protein Assay kit was purchased from Pierce Biotechnology. The enhanced chemiluminescence (ECL) Plus detection kit was purchased from GE Healthcare.

\section{Electrophysiology}

Slice preparation. Horizontal sections $(150 \mu \mathrm{m})$ containing the VTA were prepared with a vibratome in an ice-cold cutting solution containing (in mм) $40 \mathrm{NaCl}, 143.5$ sucrose, $4 \mathrm{KCl}, 1.25 \mathrm{NaH}_{2} \mathrm{PO}_{4}, 26 \mathrm{NaHCO}_{3}, 0.5$ $\mathrm{CaCl}_{2}, 7 \mathrm{MgCl}_{2}, 10$ glucose, 1 sodium ascorbate, and 3 sodium pyruvate, saturated with $95 \% \mathrm{O}_{2}$ and $5 \% \mathrm{CO}_{2}$. Slices were then incubated in the same solution at $32^{\circ} \mathrm{C}$ for $45 \mathrm{~min}$ and subsequently maintained at room temperature in the external solution containing (in $\mathrm{mm}$ ) $125 \mathrm{NaCl}, 2.5$ $\mathrm{KCl}, 2.5 \mathrm{CaCl}_{2}, 1.3 \mathrm{MgCl}_{2}, 1.25 \mathrm{NaH}_{2} \mathrm{PO}_{4}, 25 \mathrm{NaHCO}_{3}$, and 10 glucose, saturated with $95 \% \mathrm{O}_{2}$ and $5 \% \mathrm{CO}_{2}$. For recordings in slices from virusand DiI-injected animals, rats were intracardially perfused with the icecold cutting solution before sectioning. Slices were incubated at $32^{\circ} \mathrm{C}$ in the cutting solution for $25 \mathrm{~min}$ and then in a solution containing the cutting and the external solution (1:1 in volume) for an additional $25 \mathrm{~min}$ at $32^{\circ} \mathrm{C}$. Slices were maintained at room temperature in the external solution until use.

Electrophysiological recording. Individual slices were placed in a recording chamber (RC26G; Warner Instruments) and viewed with an epifluorescence Olympus microscope (BX50WI). The chamber was superfused with the external solution at a speed of $2 \mathrm{ml} / \mathrm{min}$. The temperature of the chamber solution was controlled at $33-34^{\circ} \mathrm{C}$ by a dual-channel controller (TC-344B; Warner Instruments). DiI-labeled neurons were identified by the red fluorescence of the DiI and patched under the guidance of infrared-differential interference contrast (DIC) microscopy. The electrode resistance for all experiments was $4-6 \mathrm{M} \Omega$. The pipette solution contained $123 \mathrm{~mm}$ potassium gluconate, $10 \mathrm{~mm}$ HEPES, $0.2 \mathrm{~mm}$ EGTA, 8 $\mathrm{mm} \mathrm{NaCl}, 2$ mm MgATP, 0.3 mm NaGTP, and 0.1\% biocytin, $\mathrm{pH}$ 7.2-7.3, with an osmolarity of $270-280 \mathrm{mOsm}$. For measuring the spontaneous firing of neurons, cell-attached recordings were conducted in voltage- clamp mode. Data were collected using a MultiClamp 700A amplifier controlled by the pClamp 9 software (Molecular Devices). Spontaneous spikes were filtered at $2 \mathrm{kHz}$ and digitized at $10 \mathrm{kHz}$. Neurons with wide spikes and low firing frequencies were selected for data collection. At the end of the cell-attached recording, whole-cell access was obtained for biocytin perfusion into the neuron for later $\mathrm{TH}$ staining while simultaneously recording $I_{\mathrm{h}}$ in voltage-clamp mode by a hyperpolarizing step from the holding voltage of -60 to $-90 \mathrm{mV}$ for $1 \mathrm{~s}$. We also tested the $I_{\mathrm{h}}$-mediated "Sag" in current-clamp mode (data not shown). The spike frequency was continuously measured on-line with Clampex (Molecular Devices) or afterward using Clampfit or MiniAnalysis (Synaptosoft). For electrophysiological experiments in which GDNF, adenovirus expressing GDNF shRNA (Adv-shGDNF), and their respective controls were used in vivo, the firing rates of neurons were compared in a counterbalanced manner so that the influence of uncontrollable experimental conditions on the neuronal firing were minimized. Specifically, on a single experimental day, the firing rates of VTA neurons were compared between a rat treated with GDNF or Adv-shGDNF and a rat treated with vehicle or the scrambled shRNA GDNF sequence (Adv-SCR), respectively. In addition, neurons from rats receiving treatment or control were alternately recorded. For each neuron, the firing rate during a 4 min stable recording was measured. Recordings were performed within $6 \mathrm{~h}$ after slice preparation and recovery $(\sim 1 \mathrm{~h})$.

Measurement of miniature EPSCs and miniature IPSCs. For recordings of miniature EPSCs (mEPSCs), $100 \mu \mathrm{M}$ picrotoxin and $1 \mu \mathrm{M}$ TTX were present in the bath solution. The pipette solution was the same as described above. mEPSCs were recorded for $5 \mathrm{~min}$ with neurons clamped at $-70 \mathrm{mV}$. For recordings of miniature IPSCs (mIPSCs), $10 \mu \mathrm{M}$ NBQX and $1 \mu \mathrm{M}$ TTX were present in the bath solution. The intracellular solution contained (in mM) $125 \mathrm{KCl}, 4 \mathrm{NaCl}, 10 \mathrm{HEPES}, 1$ EGTA, $1 \mathrm{MgCl}_{2}, 2$ $\mathrm{Na}_{2} \mathrm{ATP}, 0.6 \mathrm{Na}_{3} \mathrm{GTP}, 2 \mathrm{Na}_{2} \mathrm{CrPO}_{4}$, and 10 QX-314. mIPSCs were recorded for $3 \mathrm{~min}$ with neurons clamped at $-70 \mathrm{mV}$. mEPSCs and mIPSCs were analyzed with the Mini Analysis Program (Synaptosoft), with detection criteria set at $>7 \mathrm{pA}$ in amplitude and verified by eye.

Ex vivo application of GDNF. GDNF was dissolved in a bath solution containing $60 \mu \mathrm{g} / \mathrm{ml}$ bovine serum albumin. After application, the GDNF solution was recirculated. The recycled solution was aspirated by one channel of a dual-channel mini-pump (P720; Instech Lab) to a 12.5 $\mathrm{ml}$ reservoir where it was reoxygenated with $95 \% \mathrm{O}_{2}$ and $5 \% \mathrm{CO}_{2}$. The reoxygenated solution was driven by the other channel of the mini-pump to the recording chamber. A similar recirculating system was previously used to deliver another neurotrophic factor, brain-derived neurotrophic factor (BDNF), into slices for electrophysiological recording (Lauterborn et al., 2007).

Postrecording TH immunostaining was performed according to Margolis et al. (2008). Briefly, immediately after electrophysiological recordings, VTA slices containing biocytin-filled neurons were fixed in $4 \%$ paraformaldehyde for $2 \mathrm{~h}$ and stored at $4^{\circ} \mathrm{C}$ in PBS. Slices were incubated with anti-TH antibody $(1: 100)$ at $4^{\circ} \mathrm{C}$ for $48 \mathrm{~h}$ and agitated overnight at $4^{\circ} \mathrm{C}$ with Cy5 anti-rabbit secondary antibody (1:100) for TH detection and FITC-conjugated streptavidin for biocytin detection. Images were taken with an LSM 510 META microscope (Zeiss). The colors of TH and biocytin immunostaining were switched using the LSM program (Zeiss) so that $\mathrm{TH}$ was green, consistent with the color of $\mathrm{TH}$ immunostaining in other experiments (see Figs. $2 D, 4 B$ ). TH staining of the VTA from GDNF-injected (see Fig. $2 D$ ) and DiI-injected (see Fig. $4 B$ ) animals was done as described by Carnicella et al. (2008).

$\mathrm{TH}$ immunostaining stated otherwise was done as described by Carnicella et al. (2008)

\section{Terminal deoxynucleotidyl transferase-mediated biotinylated UTP nick end labeling staining}

Eighteen days after intra-NAc injection of virus, VTA sections were prepared and permeabilized with 50\% ethanol in PBS for 20 min, rinsed in PBS, incubated in terminal deoxynucleotidyl transferase-mediated biotinylated UTP nick end labeling (TUNEL) reaction mixture (In Situ cell detection kit; Roche Applied Science) for $1 \mathrm{~h}$ at $37^{\circ} \mathrm{C}$ according to the vendor's protocol, rinsed, and processed for TH immunostaining as described by Carnicella et al. (2008). 
In vivo application of GDNF and $A d v$-sh GDNF

Intra-VTA infusions. GDNF was infused into the VTA as described previously (Carnicella et al., 2008). Briefly, GDNF (10 $\mu \mathrm{g}$ in $1 \mu$ l of PBS) or vehicle (PBS) was bilaterally injected into the VTA [in mm: -4.8 anteroposterior (AP), \pm 0.75 mediolateral $(\mathrm{ML}),-7.8$ dorsoventral (DV)] of rats. VTA slices were then prepared for electrophysiological recordings $10 \mathrm{~min}$ after the injections.

Intra-NAc infusions. Rats were anesthetized with isoflurane. The tip of the injector (31 gauge; Small Parts) was stereotaxically positioned into the NAc. The injector was connected to a Hamilton syringe $(10 \mu \mathrm{l})$ that was driven by an automatic pump (Harvard Apparatus). The injection speed was $0.2 \mu \mathrm{l} / \mathrm{min}$. Infusions of GDNF ( $10 \mu \mathrm{g}$ in $2 \mu \mathrm{l}$ of saline, or $2 \mu \mathrm{l}$ of saline as vehicle) and viruses (Adv-shGDNF or Adv-SCR $\left.0.35 \times 10^{10} \mathrm{TU} / \mathrm{ml}, 2 \mu \mathrm{l}\right)$ into the NAc were made bilaterally or unilaterally (for detection of ERK1/2 phosphorylation). For dual-site injections, GDNF or vehicle (saline) was infused into the NAc 7-11 d after intraprefrontal cortex (PFC) injection of DiI (see below). Each side of the NAc received four injections, varying the $\mathrm{AP}$ and $\mathrm{DV}$ axes (in $\mathrm{mm}$ : $+1.7 \mathrm{AP} 1,+1.1 \mathrm{AP} 2, \pm 1.3 \mathrm{ML},-7.2 \mathrm{DV} 1$, $-6.8 \mathrm{DV} 2)$ to reach a maximal area in the NAc. Twelve hours after injections, coronal sections of the NAc were prepared for placement verification, coronal sections of the VTA were prepared for immunostaining of phosphoERK1/2 and TH using a procedure described previously (Carnicella et al., 2008), and horizontal sections of the VTA were used for electrophysiology recordings. For retrograde labeling of NAc-projecting VTA neurons, DiI $(0.3 \mu \mathrm{l}$, in $7 \%$ ethanol) was bilaterally injected into the NAc (in $\mathrm{mm}:+1.5 \mathrm{AP}, \pm 1.3 \mathrm{ML},-7.2$ DV). Seven to 10 days after injection, coronal sections of the NAc were prepared for thionin $(0.2 \%)$ staining to verify injection placements, coronal sections of the VTA were prepared for TH staining to examine the overlay of DiI- and TH-labeled cells, and horizontal sections of VTA were used for electrophysiology recordings.

Intra-PFC injection. DiI $(0.3 \mu \mathrm{l}, 7 \%$ in ethanol) was bilaterally injected into the medial PFC (in mm: $+2.6 \mathrm{AP}, \pm 0.78 \mathrm{ML},-4.0 \mathrm{DV}$ ).

\section{Cloning and preparation of GDNF small hairpin RNA} recombinant adenovirus

A 20-nucleotide (nt) GDNF small interfering RNA (siRNA) sequence (siGDNF), ATGTCACTGACTTGGGTCTG, was designed using the online siRNA Retriever (http://cancan.cshl.edu/RNAi_central/). This siGDNF sequence targets the coding domain of the GDNF mRNA and was used for vector-based small hairpin RNA (shRNA) expression. Two complementary oligonucleotides were synthesized as follows: $5^{\prime}$ GATCCC (20 nt, sense) TTGATATCCG (20 nt, antisense), TTTTTTCCAAA- ${ }^{\prime}$ and $3^{\prime}$-GG (20 nt antisense) AACTATAGGC (20 nt, sense) AAAAAAGGTTTTCGA- $5^{\prime}$, flanked by Bam $\mathrm{H} 1$ and HindIII residues. The paired oligonucleotides were annealed and ligated into the pRNAT-H1.1/ Shuttle (GenScript Corporation), a green fluorescent protein (GFP)containing adenoviral shuttle siRNA vector. Recombinant sequences were confirmed before subcloning into the adenoviral vector Adeno-X (Clontech). Preparation of adenoviruses was performed according to the Adeno-X Expression System 1 user manual (Clontech). The scrambled
B
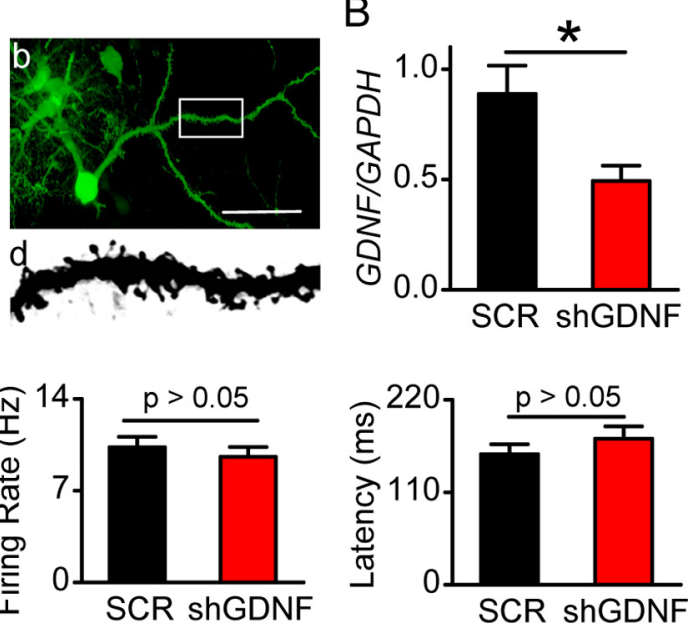

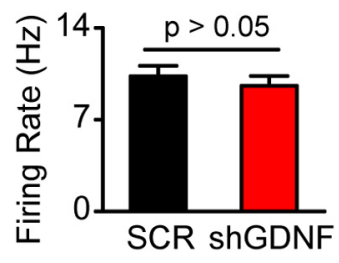

SCR
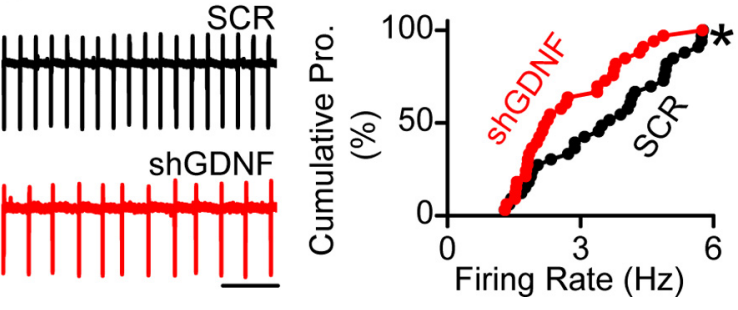

Figure 1. Viral-mediated downregulation of GDNF mRNA in the NAc does not alter evoked firing but leads to a decrease in a injection in slices from Adv-SCR (SCR; top)- and Adv-shGDNF (shGDNF; bottom)-treated rats. Calibration: 30 mV, 100 ms. Middle (middle) and latency (right) of evoked VTA neuronal firing between (shGDNF) neurons from 13 rats for each group. The latency was defined as the duration between the onset of current injection to the peak of the first spike. $n=36(\mathrm{SCR})$ and $n=35$ (shGDNF) neurons from 13 rats for each group. $D$, Downregulation of GDNF in

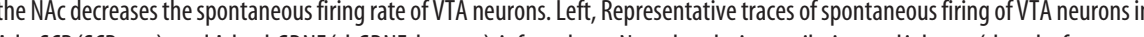
neurons in slices from Adv-shGDNF- and Adv-SCR-treated rats. ${ }^{*} p<0.05$, Kolmogorov-Smirnov test. $n=33$ neurons from 9 rats for each group. See also supplemental Figure $\$ 1$, available at www.jneurosci.org as supplemental material.

sequence of siGDNF, GTCTGGGTTCAGTCACTGTA was designed and prepared in parallel. Viruses were amplified in HEK293 cells, followed by purification using the Adeno-X Virus Purification kit (Clontech), and titered based on GFP-visualized infection. Recombinant viruses were used to infect C6 glioma cells or a stable SHSY5Y cell line that overexpresses GDNF (He and Ron, 2006) at a multiplicity of infection of 20, and downregulation of GDNF after infection was measured thereafter (supplemental Fig. S1 A, available at www.jneurosci.org as supplemental material).

\section{Brain sample collection and quantitative reverse} transcription-PCR

Eighteen days after viral infusion, brain tissue containing the NAc was dissected. Total RNA was isolated, and reverse transcription of mRNA into cDNA was conducted using the oligo $(\mathrm{dT})$ primers. The reverse transcription reaction was conducted at $42^{\circ} \mathrm{C}$ for $30 \mathrm{~min}$. The resulting cDNA samples were amplified by TaqMan quantitative PCR using commercially available primer/probe kits from Applied Biosystems for GDNF (Gene Expression 
A

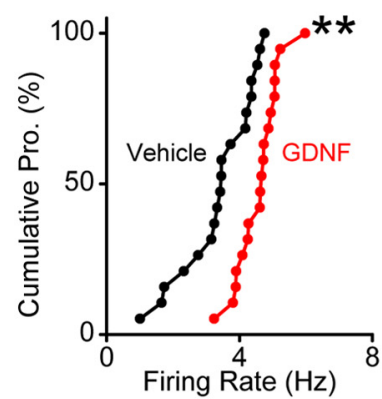

C
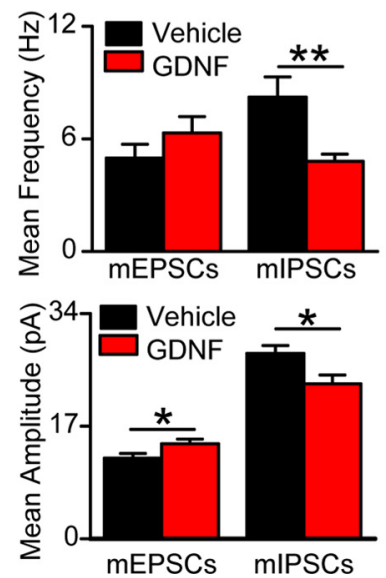

B

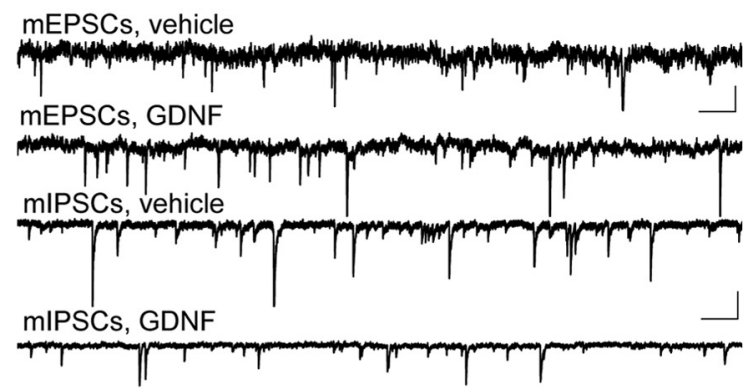

D

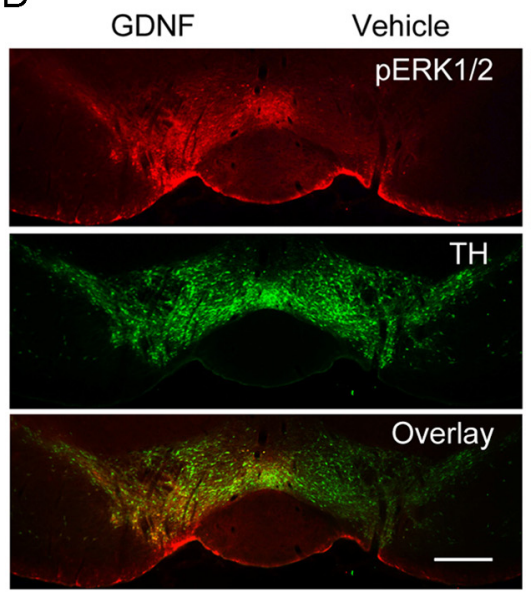

detect the level of phospho-ERK1/2, slices were pretreated with TTX $(1 \mu \mathrm{M})$ and MK-801 (20 $\mu \mathrm{M})$ for $30 \mathrm{~min}$ to block activity- and NMDA receptor-dependent changes in the level of phospho-ERK1/2. Slices were then incubated with GDNF ( $400 \mathrm{ng} / \mathrm{ml}$ ) for $45 \mathrm{~min}$ in the continuous presence of TTX and MK-801. At the end of the treatment period, the slices were quickly sonicated in radioimmunoprecipitation assay buffer ( $50 \mathrm{~mm}$ Tris- $\mathrm{HCl}, \mathrm{pH} 7.4,5$ mM EDTA, 120 mм NaCl, 1\% NP-40, 0.1\% deoxycholate, and $0.5 \%$ SDS) containing protease and phosphatase inhibitors. After a 30 min incubation of the lysate on ice, $25 \mu \mathrm{g}$ of protein was resolved in NuPAGE $4-12 \%$ BisTris gels (Invitrogen). Anti-phospho-Ret and anti-phospho-ERK1/2 antibodies (1:2000) were used to detect the phosphorylation levels of Ret and ERK1/2. ECL reaction was used for detection of signal, which was digitally scanned using a STORM detector. After detection, membranes were incubated in stripping buffer (25 mm glycine- $\mathrm{HCl}$ and $1 \% \mathrm{SDS}, \mathrm{pH} 3$, for 30 min at room temperature) and reprobed with anti-Ret or anti-ERK2 antibodies (1:1000 for Ret, 1:2000 for ERK2). Densitometric analysis was performed using ImageJ software (National Institutes of Health).

\section{Intra-NAc infusion of 6-OHDA}

Thirty minutes before 6-OHDA infusions, rats were administered with desipramine $(15 \mathrm{mg} /$ $\mathrm{kg}$, i.p.) to prevent the uptake of 6-OHDA into noradrenergic nerve endings. 6-OHDA was dissolved in saline $(8 \mu \mathrm{g} / \mu \mathrm{l})$ and ascorbate $(0.1$ $\mu \mathrm{g} / \mu \mathrm{l})$. 6-OHDA $(1.5 \mu \mathrm{l}$ ) was bilaterally (for electrophysiological recordings) or unilaterally (for immunohistochemical studies) infused into the NAc (see above for coordinates). For electrophysiology experiments, DiI was infused into the PFC (see above for coordinates) to label PFC-projecting VTA neurons. Three weeks later, GDNF $(10 \mu \mathrm{g} / 2 \mu \mathrm{l})$ or vehicle was bilaterally infused to the NAc. Twelve hours after GDNF/vehicle infusion, animals were anesthetized, and brains were removed for electrophysiological recordings or immunohistochemical studies.
Assay Rn00569510_m1) and glyceraldehyde-3-phosphate dehydrogenase (GAPDH; Gene Expression Assay Rn99999916_s1) as the internal control.

\section{Standard reverse transcription-PCR}

Five or 18 days after viral infusion, brain tissue containing the NAc or the VTA was dissected. Total RNA was isolated with TRIzol reagent and used for reverse transcription with the reverse transcription system at $42^{\circ} \mathrm{C}$ for 30 min. PCR was performed with GAPDH as an internal control, as described previously (Carnicella et al., 2009). Thirty-two cycles were performed to measure the expressions of GDNF, TH, and nerve growth factor $(N G F)$. Primers were used as follows: rat GDNF, upstream 5'-GACGTCATGGATTTTATTCAAGCCACC- $3^{\prime}$ and downstream 5'-CTGGCCTACTTTGTCACTTGTTAGCCT-3'; rat TH, upstream 5'-GAAGCTGATTGCAGAGATTGC-3' and downstream $5^{\prime}$-GCTCAGGTGAATGCATAGGTG3'; rat NGF, upstream 5'-ACACTCTGGATCTAGACTTCCAGG-3' and downstream 5' -AGGCAAGTCAGCCTCTTCTTGTAG-3'.

\section{Western blot analysis}

Midbrain slices were prepared as above. After recovery for at least $1 \mathrm{~h}$, midbrain slices $(150 \mu \mathrm{m})$ containing the VTA were dissected. To detect the phosphorylation level of Ret, slices were treated with GDNF (400 $\mathrm{ng} / \mathrm{ml}$ ) for $45 \mathrm{~min}$ at room temperature in artificial CSF (aCSF). To
In vivo microdialysis

Surgery. Adult male Long-Evans rats (350-400 g; Harlan) were anesthetized with isoflurane (Baxter Health Care Corporation) or equithesin ( $1 \%$ pentobarbital, $2 \%$ magnesium sulfate, $4 \%$ chloral hydrate, $42 \%$ propyleneglycol, $11 \%$ ethanol, and injected i.p. at $3 \mathrm{ml} / \mathrm{kg}$ ). Unilateral guide cannulae for microinjection (26 gauge; Plastics One) were placed dorsal to the VTA (in $\mathrm{mm}:-5.6 \mathrm{AP}, \pm 0.8 \mathrm{ML},-7.4 \mathrm{DV}$, relative to the bregma), and guide cannulae for the microdialysis probes (CMA/11, CMA microdialysis) were placed dorsal to the NAc (in $\mathrm{mm}:+1.7 \mathrm{AP}$, $+1.0 \mathrm{ML},-6.4 \mathrm{DV}$, relative to the bregma). After the surgery, animals were allowed to recover for $2-4 \mathrm{~d}$.

Microinjection and microdialysis procedure. Microdialysis was performed as described previously (Zapata and Shippenberg, 2005). In the evening before the experiment, probes ( $2 \mathrm{~mm}$ membrane length) were inserted into guide cannulae and connected to the dialysis system. Rats were housed in the microdialysis chamber with food and water, and the probes were perfused overnight $(0.3 \mu \mathrm{l} / \mathrm{min})$ and $1 \mathrm{~h}$ before $(1 \mu \mathrm{l} / \mathrm{min})$ experiments with aCSF (in mM: $145 \mathrm{NaCl}, 2.8 \mathrm{KCl}, 1.2 \mathrm{MgCl}_{2}, 1.2 \mathrm{CaCl}_{2}$, 0.25 ascorbic acid, and 5.4 D-glucose, $\mathrm{pH}$ 6.5-7.0 adjusted with $\mathrm{NaOH}$ ). Fresh aCSF was loaded, and probes were equilibrated over $1 \mathrm{~h}$ at a flow 
rate of $0.6 \mu \mathrm{l} / \mathrm{min}$ before the beginning of the sample collection. GDNF $(10 \mu \mathrm{g} / \mu \mathrm{l})$ or PBS and/or U0126 $(0.5 \mu \mathrm{g} / \mu \mathrm{l})$ or its vehicle $(5 \%$ DMSO and 6\% Tween 80 in PBS) was infused over $2 \mathrm{~min}$ into the VTA via injection cannulae (33 gauge; Plastics One) extending $1.0 \mathrm{~mm}$ beyond the guide cannula tip. To confirm the functional connectivity between the VTA infusion site and the NAc dialysate collection site, $2.5 \mathrm{~h}$ later, the $\mathrm{GABA}_{\mathrm{B}}$ receptor agonist baclofen $(75 \mathrm{ng} / \mu \mathrm{l})$ was infused into the VTA to inactivate DA neurons. Connectivity was verified by the resulting decrease in NAc DA concentrations. Rats showing no decrease in NAc DA levels after the infusion of baclofen were excluded from the analyses. Dialysis fractions were collected every $15 \mathrm{~min}$, frozen at $-80^{\circ} \mathrm{C}$, and analyzed for DA content within $48 \mathrm{~h}$ using HPLC electrochemical detection.

DA-level determination. DA levels in the dialysate samples of the first microdialysis experiment (effect of intra-VTA infusion of GDNF on NAc DA overflow) (see Fig. 7A) were determined as described by Zapata and Shippenberg (2005). The chromatographic system used to determine the DA levels in the dialysate samples of the second microdialysis experiment (including the intra-VTA infusion of the MAPK/ERK kinase (MEK) inhibitor U0126 (Fig. 7B) consisted of a Model 584 pump and a microtiter Model 540 (ESA), a Coulochem III amperometric detector with a Model 5011A dual-detector analytical cell (guard cell, $275 \mathrm{mV}$; E1, - $150 \mathrm{mV}$; E2, 220 $\mathrm{mV}$; ESA), and a microbore column (50 $\mathrm{mm} \times 1.5 \mathrm{~mm} \times 3 \mu \mathrm{m}$; Shizeido Company). The mobile phase $\left(150 \mathrm{~mm} \mathrm{NaH}{ }_{2} \mathrm{PO}_{4}, 4.76\right.$ mM citric acid, $50 \mu \mathrm{M}$ EDTA, $3 \mathrm{~mm}$ SDS, $8 \%$ methanol, and $10 \%$ acetonitrile, pH 5.6 adjusted with $\mathrm{NaOH}$ ) was run at a flow rate of $0.2 \mathrm{ml} / \mathrm{min}$. DA was quantified by comparing DA peaks from dialysate samples with external standards. Under these conditions, the retention time for DA was $3 \mathrm{~min}$, and the limit of detection was below $0.3 \mathrm{~nm}$ (at a signal-to-noise ratio of $3: 1$ ).

Histology. Locations of cannulae and microdialysis probe placements were verified in 30- $\mu \mathrm{m}$-thick coronal slices after cryostat sectioning, and subjects with misplaced injectors or probes were excluded from the analyses.

\section{Statistical analysis}

All values are expressed as mean \pm SEM, unless stated otherwise. Statistical significance of electrophysiological and biochemical data was analyzed by paired or unpaired Student's $t$ test unless stated otherwise. The microdialysis data were analyzed by a two-way ANOVA (mixed withinsubject design). Significant interaction was further investigated by using the method of contrasts or the Student-Newman-Keuls test.

\section{Results}

Downregulation of GDNF expression in the NAc decreases spontaneous but not evoked firing frequency of VTA neurons First, we tested whether endogenous GDNF in the NAc regulates the evoked firing activity of VTA DA neurons. To do so, we used viral-mediated gene delivery to downregulate the expression level of GDNF in the NAc and examined the consequence of GDNF knockdown on the level of evoked firing activity of DA neurons in the VTA. An adenovirus expressing shRNA against GDNF mRNA (Adv-shGDNF) (supplemental Fig. S1A, available at
B

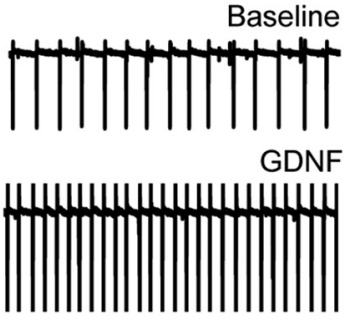

$\mathrm{E}$

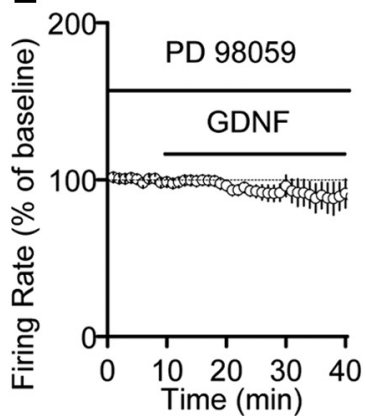

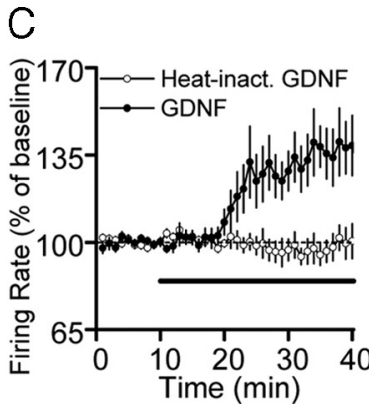

F

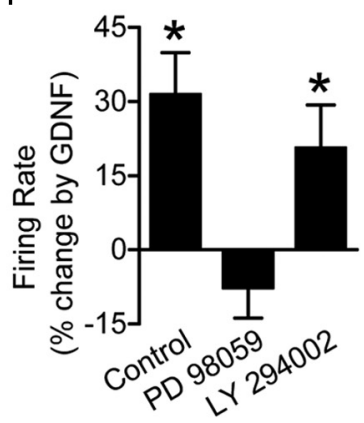

Figure 3. GDNF in the VTA increases the spontaneous firing rate of DA neurons in a MAPK-dependent manner. $\boldsymbol{A}$, Intra-VTA infusion of GDNF produces an increase in the spontaneous firing rate of VTA neurons. GDNF ( $10 \mu \mathrm{g} / \mu \mathrm{l}$ ) or vehicle was bilaterally 列 $n=12$ cells from 3 rats for each group. $\boldsymbol{B}-\boldsymbol{D}$, Bath-applied GDNF increases the spontaneous firing frequency of DA neurons. $\boldsymbol{B}$, fGDNF $(200 \mathrm{ng} / \mathrm{ml})$. Scale bar, $0.5 \mathrm{~s}$. C, Averaged time course showing that bath application of GDNF $(200 \mathrm{ng} / \mathrm{ml}$; filled circles; $n=$ (Heat-inact.; GDNF, $200 \mathrm{ng} / \mathrm{ml}$; open circles; $n=8$ ), induced an increase in the firing was heated at $95^{\circ} \mathrm{C}$ for 1 h. D Dose-response of GDNF-induced enhancement of the firing rate ${ }^{*} p<0.05$ versus baseline $n=10$ , for 50,100, and $200 \mathrm{ng} / \mathrm{ml}$ GDNF, respectively. $\boldsymbol{E}, \boldsymbol{F}$, GDNF enhancement of the spontaneous firing rate of VTA neurons (25 Fing rate of neurons in the presence of PD 98059 before and during GDNF application. $\boldsymbol{F}$, Bar graph summarizing the effect of PD 98059 and LY 294002 on the firing rate of neurons in response to GDNF. ${ }^{*} p<0.05$ versus baseline. $n=9$ (PD 98059) and $n=11$ See also supplemental Figure $\$ 3$, available at www.jneurosci.org as supplemental material.

www.jneurosci.org as supplemental material) was stereotaxically and bilaterally infused into the NAc of rats (Fig. $1 A a$ ). Intra-NAc administration of Adv-shGDNF led to infection of medium spiny neurons (MSNs) (Fig. 1 $A b-A d$ ), which are the principal neurons of the NAc that produce GDNF (Oo et al., 2005). Infection of neurons with Adv-shGDNF produced an $\sim 50 \%$ reduction in GDNF mRNA levels in the NAc $18 \mathrm{~d}$ after infusion of the virus (Fig. $1 B$; supplemental S1 $B$, available at www.jneurosci.org as supplemental material). The knockdown of GDNF expression was time dependent (supplemental Fig. S1 B, available at www.jneurosci.org as supplemental material) and specific, as the mRNA level of another growth factor, $N G F$, was unaltered (supplemental Fig. S1 B, available at www. jneurosci.org as supplemental material).

Next, we determined whether the downregulation of GDNF expression in the NAc alters the evoked firing of neurons in the VTA, $18 \mathrm{~d}$ after viral infusion. To do so, we used whole-cell current-clamp recordings, in which a depolarizing step current of $120 \mathrm{pA}$ was injected to induce firing with the first two-spike frequency of $10 \mathrm{~Hz}$ (Fig. 1C, left) as described previously (Yang et al., 2001). No differences in firing frequency (Fig. 1C, middle) or latency to the first spike (Fig. $1 C$, right) were observed between neurons from rats infected with Adv-shGDNF and rats infected 
A

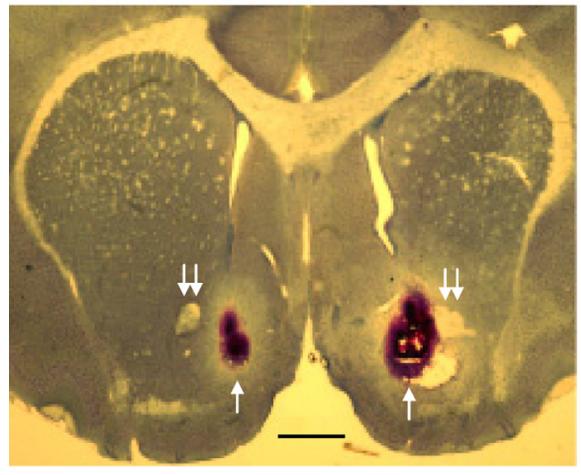

B

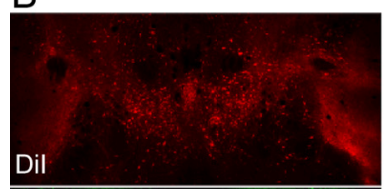

Dil

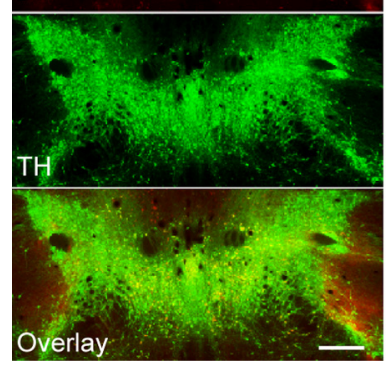

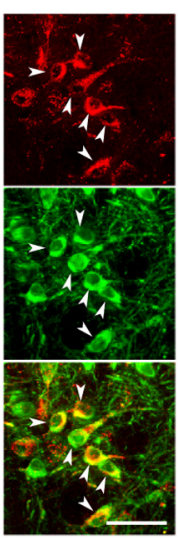

C
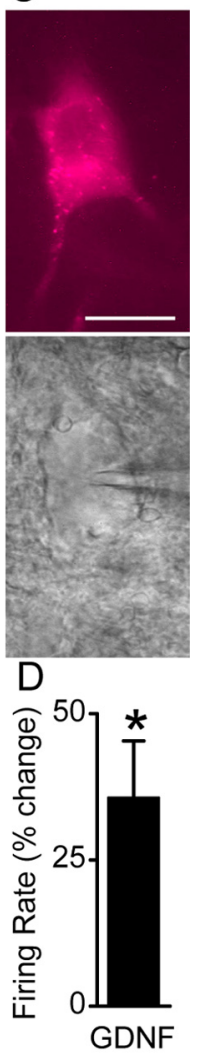

Figure 4. GDNF increases the firing of the NAc-projecting DA neurons in the VTA. The retrograde tracer Neuro-Dil was injected bilaterally into the NAC. $A$, Representative coronal section confirming the injection sites within the NAc. Arrows indicate tracer deposit. The double arrows indicate the anterior commissure. Scale bar, $1 \mathrm{~mm}$. B, Representative images showing that numerous Neuro-Dil-labeled VTA neurons are also TH positive. Shown are dual-channel fluorescent images for Dil (red), TH (green), and overlay (yellow). The arrowheads indicate cells labeled with Dil and stained for TH (right). Scale bars: left, $500 \mu \mathrm{m}$; right, $50 \mu \mathrm{m}$. C, Representative images showing a Dil-labeled VTA neuron that was selected for electrophysiology. Top, Red fluorescent image. Bottom, DIC image. Scale bar, $20 \mu \mathrm{m}$. D, Bar graph summarizing the mean increase by GDNF $(200 \mathrm{ng} / \mathrm{ml})$ in the firing rate of Neuro-Dillabeled VTA neurons. ${ }^{*} p<0.05 . n=8$ cells.
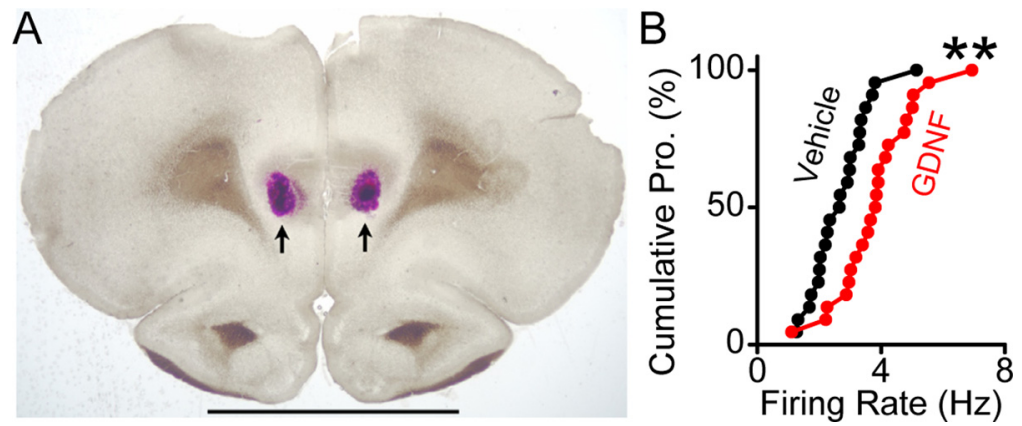

Figure 5. Intra-NAc infusion of GDNF leads to an increase in spontaneous firing rate of PFC-projecting VTA neurons. GDNF (10 $\mu \mathrm{g} / 2 \mu \mathrm{l})$ was bilaterally infused into the NAc 7-11 d after intra-PFC infusions of Dil. VTA slices were prepared $12 \mathrm{~h}$ after GDNF infusion, and the spontaneous firing of Dil-labled PFC-projecting neurons was measured. $\boldsymbol{A}$, Representative coronal section confirming the injection sites within the PFC. Arrows indicate Dil deposit. Scale bar, $0.5 \mathrm{~mm}$. B, Cumulative probability (Cumulative Pro.) plot comparing spontaneous firing rates of individual neurons in slices from vehicle-treated (black circles) and GDNF-treated (red circles) srats. ${ }^{* *} p<0.01$ versus vehicle, Kolmogorov-Smirnov test. $n=22$ cells from 5 rats for each group.

with a control adenovirus expressing the Adv-SCR. These results suggest that endogenous GDNF in the NAc does not regulate the evoked firing of VTA neurons.

DA neurons also exhibit spontaneous tonic firing (Grace and Bunney, 1984). Therefore, using cell-attached recordings (Fig.
$1 D$, left), we compared the spontaneous firing rate of VTA neurons from Adv-SCR- and Adv-shGDNF-infected rats. Interestingly, we observed that the spontaneous firing rate of VTA neurons was markedly decreased in the Adv-shGDNF-treated rats compared with those treated with Adv-SCR (Fig. 1D) (shGDNF, $2.74 \pm 0.21 \mathrm{~Hz}$; SCR, $3.57 \pm 0.26 \mathrm{~Hz} ; p<0.05, t$ test).

Pascual et al. (2008) recently reported that conditional knockout of GDNF in adult mice leads to a loss of TH mRNA and to a decrease in the number of DA neurons in the VTA 3 and 7 months after the onset of the knock-out, respectively. We therefore tested whether knockdown of GDNF in the NAc resulted in cell death of VTA DA neurons. We found that the short-term (18 d), 50\% reduction in the GDNF level in the NAc did not produce a change in the mRNA level of TH in the VTA (supplemental Fig. S1C, available at www.jneurosci.org as supplemental material), or in the number of TH-immunoreactive neurons (supplemental Fig. S1 $D$, available at www.jneurosci.org as supplemental material), and no significant neuronal cell death was detected in the VTA as measured by TUNEL staining (supplemental Fig. S1 $D$, available at www.jneurosci.org as supplemental material). These results indicate that attenuation of the spontaneous firing rate of VTA neurons by downregulation of NAc GDNF levels is unlikely to be attributed to cell death of VTA DA neurons. Together, these results suggest that NAc-produced endogenous GDNF is required for maintaining the normal spontaneous firing activity of VTA neurons.

Intra-NAc application of GDNF activates the MAPK pathway and increases neuronal firing in the VTA

If GDNF, produced in the NAc, is transported to the VTA to regulate the spontaneous firing rate of DA neurons, then infusion of recombinant GDNF into the NAc of rats should increase the spontaneous firing activity of VTA neurons. To test this possibility, we bilaterally infused GDNF or vehicle into the NAc of rats and measured the spontaneous activity of VTA neurons in slices prepared $12 \mathrm{~h}$ after injection. We found that the spontaneous firing rate of VTA neurons was higher in rats that received intra-NAc infusion of GDNF than in rats that were infused with vehicle (Fig. 2A) (GDNF, $4.84 \pm$ $0.28 \mathrm{~Hz}$; vehicle, $3.16 \pm 0.28 \mathrm{~Hz} ; p<$ $0.01, t$ test).

Unlike the evoked firing activity that is determined primarily by the intrinsic excitability of neurons (Yang et al., 2001; Hahn et al., 2003), the spontaneous firing activity is controlled by both the intrinsic excitability (Liss et al., 2001; Khaliq and Bean, 2010) and the extrinsic synaptic drive (Johnson et al., 1992). To test whether the distinct effect of NAc-derived GDNF on the spontaneous, but not on the evoked, firing rates results from changes in synaptic drive, we measured the level of mEPSCs and mIPSCs of VTA neurons $12 \mathrm{~h}$ after intra-NAc infusion of GDNF. We found that GDNF treatment causes a small but significant increase in the amplitude, but not in the frequency, of mEPSCs (Fig. $2 B, C$ ). In addition, we observed that GDNF application leads to decreases in both the amplitude and the frequency of mIPSCs (Fig. $2 B, C$ ). These results suggest that NAc-derived GDNF regulates the extrinsic synaptic drive (i.e., 
increases the excitatory, but decreases the inhibitory, synaptic strength to the neurons).

The GDNF receptors GFR $\alpha 1$ and RET are highly expressed in the VTA (Trupp et al., 1997). After receptor ligation, GDNF activates several signaling cascades including the MAPK, ERK1/2mediated pathway (Airaksinen and Saarma, 2002). Therefore, we tested whether intra-NAc infusion of GDNF also leads to the activation of the ERK1/2 in the VTA. GDNF was infused into the NAc of one hemisphere, and vehicle was injected into the other hemisphere (supplemental Fig. S2, available at www.jneurosci.org as supplemental material), and the activation of ERK1/2 was assessed by measuring the phosphorylation level of the enzyme in VTA slices, $12 \mathrm{~h}$ after infusion. We found that the level of phospho-ERK1/2 in DA neurons was significantly higher in the side ipsilateral to the intra-NAc infusion of GDNF compared with that of the vehicle infusion (Fig. 2D). Together, these results suggest that NAc-derived GDNF activates the MAPK pathway and increases the spontaneous firing rate of VTA DA neurons.

\section{GDNF enhancement of VTA neuronal firing requires the activation of the MAPK pathway}

If GDNF in the NAc is transported to the VTA to regulate the spontaneous firing of VTA neurons via ERK1/2, then the activation of the GDNF pathway directly in the VTA should produce the same response. First, we tested whether intra-VTA infusion of GDNF in vivo alters the spontaneous firing activity of VTA neurons. To do so, GDNF or vehicle was infused bilaterally into the VTA of rats, slices were prepared $10 \mathrm{~min}$ after infusion, and the spontaneous firing activity of neurons was measured after slice recovery. As shown in Figure $3 A$, intra-VTA application of GDNF produced an increase in the spontaneous firing frequency of VTA neurons.

Next, we tested whether the activation of the MAPK pathway is required for GDNF-mediated increase in spontaneous activity of VTA neurons. As shown in Figure $3 B-D$, bath application of GDNF, but not heat-inactivated GDNF, induced a dosedependent and rapid increase in the spontaneous firing rate of VTA neurons that exhibited large hyperpolarization-activated cation currents $\left(I_{\mathrm{h}}\right)$ and were TH positive (supplemental Fig. $\mathrm{S} 3 A, B$, available at www.jneurosci.org as supplemental material) but did not alter the activity of neurons that were small and TH negative (supplemental Fig. S3C-E, available at www. jneurosci.org as supplemental material). Next, we confirmed that application of GDNF leads to the activation of the Ret receptor and to the activation of ERK $1 / 2$ in midbrain slices (supplemental Fig. S3F, G, available at www.jneurosci.org as supplemental material). Finally, we determined the contribution of ERK1/2 to the GDNF-mediated increase in the spontaneous firing rate of VTA DA neurons, by measuring the effects on GDNF on the spontaneous firing rate of neurons in the presence of PD 98059, a specific inhibitor of the MEK that phosphorylates and activates ERK1/2. PD 98059 did not alter the activity of VTA neurons (supplemental Fig. S3H, available at www.jneurosci.org as supplemental material), and GDNF failed to induce an increase in the firing rate of VTA DA neurons in slices pretreated with the inhibitor (Fig. $3 E, F$ ). In contrast, blockade of the PI3K pathway with the specific inhibitor LY 294002 did not alter the GDNF-mediated increase in the level of spontaneous firing of VTA DA neurons (Fig. $3 F)$. Together, these results suggest that GDNF in the VTA increases the spontaneous firing rate of VTA DA neurons via the activation of the MAPK pathway.
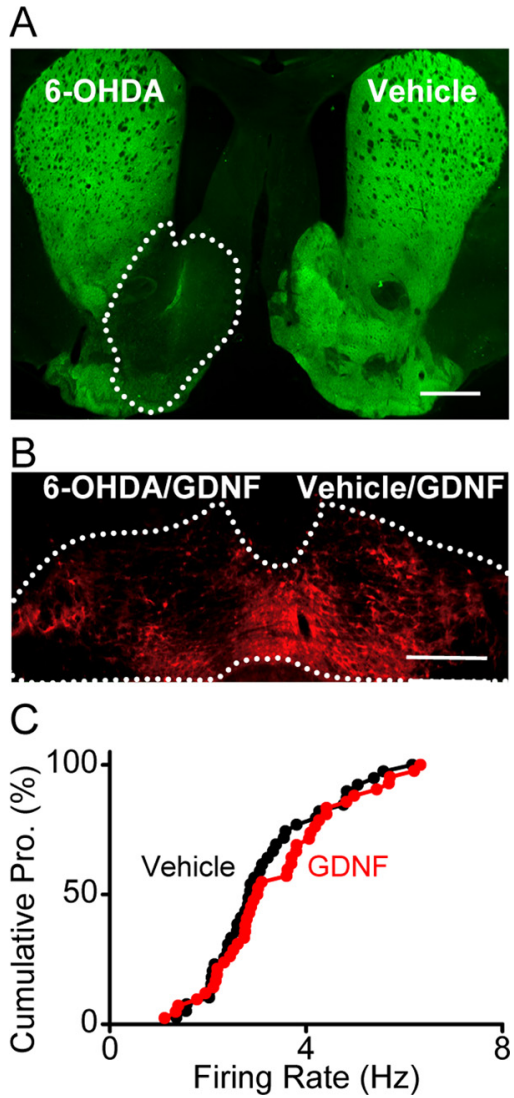

Figure 6. 6-OHDA-mediated lesions of DAergic fibers in the NAC attenuates NAc GDNFinduced activation of VTA ERK1/2 and enhancement of the spontaneous firing rate of PFCprojecting VTA neurons. 6-0HDA was unilaterally $(\boldsymbol{A}, \boldsymbol{B})$ or bilaterally $(\boldsymbol{C})$ infused into the NAC. For electrophysiology experiments ( $\boldsymbol{C}$, Dil was also bilaterally infused into the PFC. Three weeks after the infusion of 6-OHDA, vehicle $(\boldsymbol{A})$ or GDNF $(10 \mu \mathrm{g} / 2 \mu \mathrm{l} ; \boldsymbol{B}, \boldsymbol{C})$ was bilaterally infused into the NAc. Twelve hours after GDNF infusion, animals were perfused, and brains were removed to examine the immunoreactivity of TH $(\boldsymbol{A})$ and p-ERK1/2 $(\boldsymbol{B})$. The spontaneous firing of Dillabeled, PFC-projecting VTA neurons was measured in parallel (C). $\boldsymbol{A}$, Image depicts TH staining in the whole striatum after 6-0HDA (left) and vehicle (right) infusion into the NAc. The reduction of TH level in the area defined by the dashed line contains the majority of the NAc. Scale bar, 1 $\mathrm{mm}$. $\boldsymbol{B}$, Intra-NAc infusion of 6-OHDA reduces subsequent GDNF-mediated increase in p-ERK1/2 levels in the VTA. The image shows ERK1/2 phosphorylation in the VTA after unilateral 6-OHDA (left) and subsequent bilateral GDNF infusions into the NAc. Scale bar, $500 \mu \mathrm{m}$. C, 6-0HDA lesion of DAergic fibers in the NAc abolishes NAc-derived GDNF enhancement of the spontaneous firing rate of PFC-projecting VTA neurons. The cumulative probability plot comparing the firing rate of neurons from GDNF (red)- and vehicle (black)-infused animals is shown. $n=39$ (vehicle) and $n=42$ (GDNF). $p>0.05$, Kolmogorov-Smirnov test.

\section{GDNF increases the spontaneous firing rate of NAc- and} PFC-projecting VTA DA neurons

The VTA contains neurons that project to the NAc, a brain region that produces GDNF. We therefore examined whether GDNF alters the spontaneous firing rate of VTA neurons that project to the NAc. To do so, we injected the retrograde tracer, Neuro-DiI, into the NAc (Fig. $4 A$ ); the tracer was retrogradely transported to the cell bodies in the VTA (Fig. $4 B$ ). As shown in Figure $4 B$, most of the DiI-labeled neurons are TH positive (i.e., DA neurons). We then tested the effect of GDNF on the firing of DiI-labeled NAc-projecting neurons (Fig. 4C) in VTA slices and found that bath application of GDNF induced an increase in the spontaneous firing frequency of VTA neurons projecting to the NAc (Fig. $4 D$ ). These results suggest that GDNF increases the spontaneous firing rate of NAc-projecting DA neurons. 
A

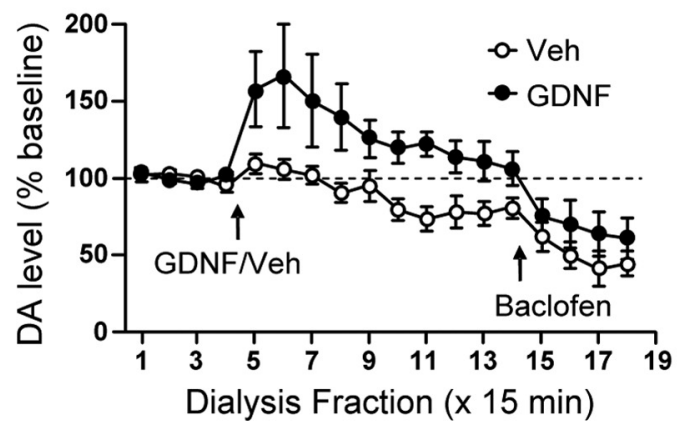

B

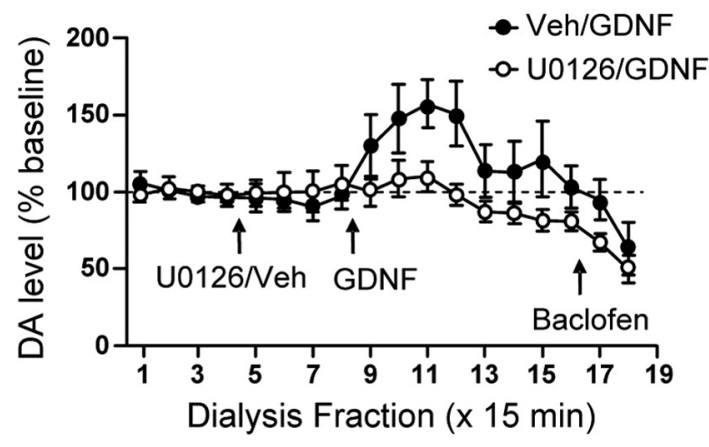

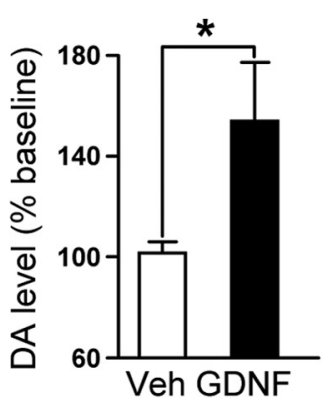

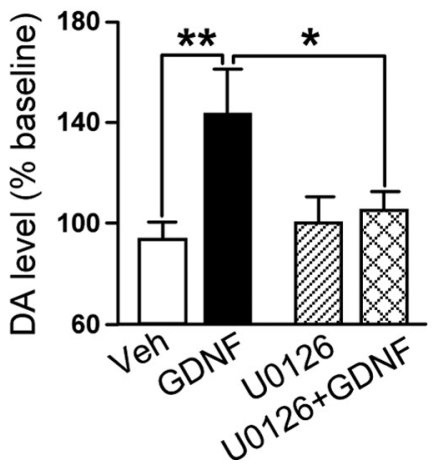

Figure 7. Application of GDNF into the VTA causes a rapid and sustained elevation of DA overflow in the NAc that is blocked by inhibition of the MAPK pathway. $A$, Left, Time course of dialysate concentrations of DA from the NAc before and after intra-VTA infusion of $10 \mu \mathrm{g} / \mu \mathrm{l}$ per side (filled circles) or vehicle (Veh; open circles). Two-way ANOVA (treatment $\times$ fractions) shows a significant effect of treatment $\left(F_{(1,156)}=9.40 ; p<0.01\right)$ and fraction $\left(F_{(13,159)}=4.88 ; p<0.001\right)$ and a significant interaction between both factors $\left(F_{(13,159)}=2.75 ; p<0.01\right)$. Post hoc analysis using the method of contrasts shows a significant difference between the vehicle and the GDNF conditions from fractions 5 to $14(T>1.81 ; p<0.05)$. The basal concentration of DA in dialysate was $1.97 \pm 0.53$ and $2.14 \pm 0.42 \mathrm{~nm}$ for the Veh and GDNF groups, respectively. Right, Bar graph comparing the DA levels in the NAc after GDNF or vehicle injections into the VTA. The values were averaged from fractions 5 to $8 . n=8$ (Veh) and $n=6$ (GDNF). $B$, Left, MEK inhibitor U0126 $(0.5 \mu \mathrm{g} / \mu$ l per side) or vehicle were infused in the VTA $1 \mathrm{~h}$ before the application of GDNF $(10 \mu \mathrm{g} / \mu \mathrm{l}$ per side) or vehicle. The time course of dialysate concentrations of DA from the NAc before and after intra-VTA infusion of GDNF (filled circles) or GDNF/U0126 (open circles) is shown. Two-way ANOVA (treatment $X$ fractions) shows a significant effect of treatment $\left(F_{(1,156)}=8.35 ; p<0.01\right)$ and fraction $\left(F_{(13,159)}=4.03 ; p<0.001\right)$ and no interaction between both factors $\left(F_{(13,159)}=1.86\right.$; $p=0.12$ ). Post hoc analysis using the method of contrasts shows a significant difference between the Veh/GDNF and the U0126/ GDNF conditions from fractions $11,12,15$, and $17(t>1.68 ; p<0.05)$. The basal concentration of DA in dialysate was $0.75 \pm 0.18$ and $1.13 \pm 0.15 \mathrm{~nm}$ for the Veh/GDNF and U0126/GDNF groups, respectively. Right, Bar graph comparing the DA levels in the NAC after vehicle, U0126, or GDNF injections into the VTA. The values were averaged from fractions 5 to 8 for the vehicle and U0126 injections and from fractions 9 to 12 for the GDNF injections. $n=6$ (Veh/GDNF) and $n=8$ (U0129/GDNF). In $\boldsymbol{A}$ and $\boldsymbol{B}$, intra-VTA infusion of $75 \mathrm{ng}$ of baclofen confirmed the functional connection between the VTA and NAc placements by reducing NAC DA overflow in the four groups. ${ }^{*} p<0.05 ;{ }^{* *} p<0.01$.

In addition to projecting to the NAc, VTA DA neurons also project to the PFC (Swanson, 1982). Although individual VTA DA neurons do not project to both the NAc and the PFC (Swanson, 1982), NAc- and PFC-projecting VTA neurons are located in close proximity to one another (Lammel et al., 2008; Margolis et al., 2008). We were interested in examining whether intra-NAc application of GDNF alters the spontaneous firing activity of PFC-projecting neurons in VTA slices. Specifically, we tested whether NAc-applied GDNF, which after transport is released in the VTA, can diffuse and bind to its receptors on the neighboring VTA neurons that project to the PFC, leading to alterations in firing activity of the PFC-projecting neurons. To address this possibility, we first bilaterally injected DiI into the PFC of rats to label PFC-projecting VTA neurons (Fig. 5A). Next, GDNF or vehicle was infused into the NAc 7-11 d after DiI injection, VTA slices were prepared $12 \mathrm{~h}$ later, and the level of the spontaneous firing of PFC-projecting VTA neurons was mea-

sured. As shown in Figure 5B, the spontaneous firing frequency of PFC-projecting neurons was higher in GDNF-treated rats than in those treated with vehicle (GDNF, $3.83 \pm 0.27 \mathrm{~Hz}$; vehicle, $2.69 \pm 0.20 \mathrm{~Hz}$; $p<0.01, t$ test). These results suggest that NAc-produced GDNF is transported to the VTA where the growth factor acts to increase the spontaneous firing activity of both NAc- and PFC-projecting VTA neurons.

\section{Retrograde transport is required for} NAc-derived GDNF to enhance the spontaneous firing rate of mesocortical DA neurons

It is possible that GDNF is transported from the NAc to the VTA either retrogradely by the NAc-projecting DA neurons and/or anterogradely by the MSNs, some of which were found to project to the VTA (Lu et al., 1998). To determine whether the retrograde transport of GDNF is required for NAc GDNFmediated enhancement of firing activity of VTA neurons, we infused 6-OHDA into the NAc to selectively lesion NAcprojecting DA neurons (Joyce et al., 1983) and examined whether subsequent intraNAc infusion of GDNF still induces an increase in the firing activity of VTA PFCprojecting neurons. We found that 6-OHDA application leads to a significant reduction of $\mathrm{TH}$-positive fibers in the NAc (Fig. 6A). Importantly, after infusion of GDNF into the NAc, we observed a robust reduction in both the activation of ERK1/2 (Fig. 6B) and in the spontaneous firing rate of VTA PFC-projecting neurons (Fig. 6C) (vehicle, $3.17 \pm 0.19 \mathrm{~Hz}$; GDNF, $3.38 \pm 0.20 \mathrm{~Hz} ; p>0.05, t$ test $)$. Together, these results support the notion that the retrograde transport of GDNF is required for NAc-derived GDNF to enhance the firing activity of VTA neurons.

Infusion of GDNF into the VTA increases DA release in the NAc via the activation of the MAPK pathway

Finally, we set out to determine a consequence of the GDNFmediated enhancement of the spontaneous firing frequency of VTA DA neurons. Spontaneous firing activity of DA neurons in the midbrain leads to tonic release of DA in the target regions (Keefe et al., 1993). We therefore tested whether the rapid GDNFmediated enhancement of firing activity of DA neurons would cause a rapid increase in DA levels in the NAc using microdialysis in freely moving rats. Since the microdialysis experiment was done in adult rats and the above electrophysiology experiments was done in young rats, we first confirmed that GDNF also increases the spontaneous firing rate in VTA slices from adult rats that were the same age as those used in microdialysis (supplemental Fig. S4, available at www.jneurosci.org as supplemental material). Next, we measured DA overflow in the NAc before and 


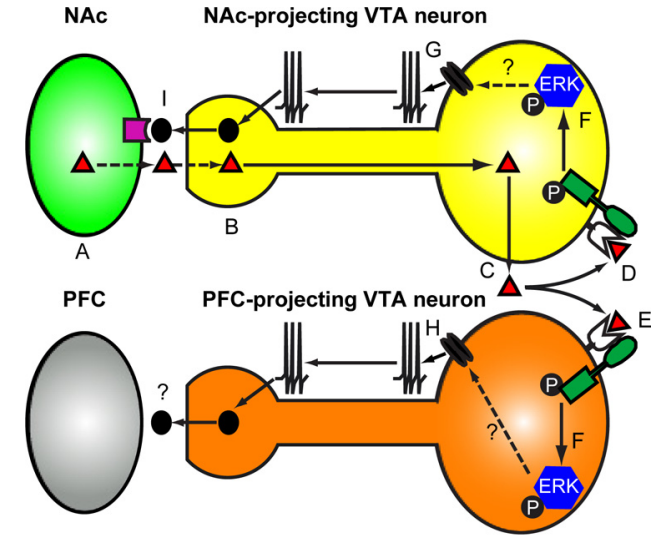

$\triangle G D N F$ שRet Dopamine

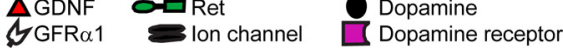

Figure 8. Model for GDNF production, transportation, and regulation of VTA neuronal activity. A VTA DA neuron (yellow) innervates an MSN (green), the principal cell of the NAc. A neighboring VTA DA neuron (orange) projects to the PFC (gray). $A$, GDNF (red triangle) is synthesized in and released by the MSN. $\boldsymbol{B}$, The polypeptide is taken up by the terminal of the NAc-projecting VTA DA neuron. C, GDNF is then retrogradely transported into the neuronal soma and/or dendrite located in the VTA, where GDNF is secreted. $\boldsymbol{D}, \boldsymbol{E}$, The released GDNF binds to GFR $\alpha-1$ (white) localized on the membrane of the same and/or adjacent NAc-projecting $(\boldsymbol{D})$ and PFC-projecting (E) VTA neuron, which leads to the ligation of the GDNF/GFR $\alpha$-1 complex to Ret (green), leading to its activation. $F-I$, Activation of Ret results in the activation of ERK1/2 (blue; $\boldsymbol{F}$ ) that, in turn, causes increased spontaneous neuronal firing $(\boldsymbol{G}, \boldsymbol{H})$, which propagates along the axon back to the DA terminal in the NAc, leading to the elevation of DA (black) release $(I)$.

after intra-VTA infusion of GDNF. We found that DA overflow in the NAc was increased shortly after infusion of the growth factor into the VTA (Fig. 7). Subsequent infusion of the $\mathrm{GABA}_{B}$ receptor agonist baclofen decreased DA levels in both vehicleand GDNF-infused rats (Fig. 7, left), confirming the functional connectivity between the VTA infusion site and the NAc dialysate collection site. Importantly, GDNF failed to elevate DA outflow in the NAc after infusion of the MEK inhibitor U0126 into the VTA (Fig. 7B), suggesting that GDNF in the VTA increases the NAc DA release in a MAPK-dependent manner.

\section{Discussion}

In this study, we show that downregulation of GDNF expression in the NAc by RNA interference significantly reduces spontaneous firing activity of VTA DA neurons. We observed that infusion of recombinant GDNF into the NAc mimics the actions of endogenous GDNF by increasing the spontaneous firing activity of VTA neurons. In addition, we show that the spontaneous firing activity of PFC-projecting VTA neurons is increased by intraNAc application of GDNF and report that GDNF in the VTA produces a rapid increase in DA release in the NAc. Finally, we present data to suggest that the increase in the spontaneous firing rate of VTA DA neurons and the increase in DA overflow in the NAc depend on the activation of ERK1/2. Based on these results, we propose the following model: GDNF produced in the target region (the NAc) (Fig. 8A) is retrogradely transported to its site of action (the VTA) (Fig. $8 B, C$ ) where the growth factor activates the GDNF receptor Ret (Fig. $8 D, E$ ), leading to the consequent activation of the MAPK signaling cascade (Fig. $8 F$ ), which, in turn, leads to a rapid enhancement of the spontaneous firing rate of both NAc- and PFC-projecting DA neurons in the VTA (Fig. $8 G, H)$. We also provide evidence that the GDNF-mediated increases in the spontaneous activity of NAc-projecting VTA neu- rons results in an elevation of DA levels in the NAc (the target region) (Fig. 8I). Together, our results suggest that GDNF is a long-distance retrograde enhancer of the mesocorticolimbic DAergic system.

\section{GDNF in the NAc enhances the spontaneous firing rate of VTA neurons}

Spontaneous firing activity of neurons is controlled by both the intrinsic excitability (Liss et al., 2001; Khaliq and Bean, 2010) and the extrinsic synaptic drive to neurons (Johnson et al., 1992), whereas the evoked firing is determined primarily by the intrinsic excitability (Yang et al., 2001; Hahn et al., 2003). We observed that downregulation of GDNF in the NAc results in a decrease in the spontaneous but not the evoked firing of VTA neurons. Conversely, we found that intra-NAc application of GDNF lead to an increase in the spontaneous firing activity of VTA neurons. We also showed that intra-NAc application of GDNF resulted in an increase in mEPSC amplitude but a decrease in mIPSC frequency and amplitude of VTA neurons. These results suggest that GDNF regulates the extrinsic synaptic strength of VTA neurons. These findings may provide an explanation to the observation that alteration in evoked firing of VTA neurons was not observed after downregulation of the endogenous growth factor in the NAc. Although our data imply that GDNF in the mesolimbic system does not influence evoked firing, we cannot exclude the possibility that GDNF modulates both the spontaneous and evoked firing activity by two fundamentally different mechanisms, which depend on the activity state of the mesolimbic system.

We also show that intra-VTA infusion of GDNF causes a rapid increase in the spontaneous firing rate of neurons in the VTA and in DA release in the NAc, and therefore it is highly likely that this rapid action of GDNF is localized within the VTA. However, it is possible that NAc-produced GDNF exhibit other roles in addition to the potentiation of the spontaneous firing rate of VTA DA neurons. For example, GDNF was reported to increase the phosphorylation of $\mathrm{TH}$ in primary cultures of rat midbrain neurons (Kobori et al., 2004), which may contribute to the prolonged upregulation of DA transmission.

\section{GDNF regulates synaptic activity via a long-distance retrograde transport mechanism}

Recent studies suggest that synaptic transmission is bidirectional: for example, in addition to presynaptic to postsynaptic neurotransmission, postsynaptic cells release molecules that retrogradely regulate presynaptic neuronal activity (Regehr et al., 2009). For example, endocannabinoids are produced in postsynaptic neurons and diffuse to the presynaptic terminals, where they act locally to reduce GABA release. In addition, the postsynaptically secreted BDNF was shown to act on presynaptic TrkB receptors, which, in turn, increases presynaptic glutamate release (Zhang and Poo, 2002; Magby et al., 2006). Our results suggest that GDNF is retrogradely transported from the soma and/or dendrites of neurons in the target region (the NAc) to the cell bodies of neurons in the VTA to regulate neuronal activity and neurotransmitter release. GDNF's localization and action fit with some of the criteria for a retrograde modulator of neuronal activity (Regehr et al., 2009). GDNF is mainly synthesized and released in the postsynaptic neurons, the MSNs in the NAc (Oo et al., 2005) but not in the VTA (Trupp et al., 1997). In addition, GDNF was reported to be retrogradely transported from the striatum to the SN (Tomac et al., 1995b). Furthermore, we found that GDNF alters the activity of VTA DA neurons that project to both the NAc and the PFC. In support of the retrograde actions of 
GDNF, we show that the 6-OHDA lesion of NAc-projecting DAergic fibers abolishes the NAc-derived, GDNF-induced enhancement of PFC-projecting neurons, suggesting that the retrograde transport of the growth factor by the mesolimbic DA neurons is required for the enhancement of firing activity of VTA neurons. Individual VTA DA neurons do not project to more than one target area (Swanson, 1982). Therefore, our results suggest that NAc-derived GDNF is released in the VTA to act on the GDNF receptors on the surface of VTA PFC-projecting neurons (Fig. 8). Indeed, the close proximity of the NAc- and PFCprojecting DA neurons makes such regulation possible. Specifically, the somata of NAc- and PFC-projecting VTA neurons were found to exhibit similar distribution patterns in rats (Margolis et al., 2008) and to be adjacent to each other $(<100 \mu \mathrm{m}$ apart $)$ in mice (Lammel et al., 2008). We cannot exclude other possibilities, such as GDNF acting indirectly on local interneurons or synapses to regulate the spontaneous firing of PFC-projecting VTA neurons. Nevertheless, our results suggest that GDNF that is transported by a single DA neuron may affect the spontaneous firing rate of a group of neighboring DA neurons that project to several other brain regions.

A possible molecular mechanism underlying GDNF-mediated enhancement in spontaneous neuronal firing rate in the VTA We observed that GDNF increases the activity of VTA DA neurons, but not non-DA neurons, and these findings are in line with the observations that GFR $\alpha 1$ is preferentially (Sarabi et al., 2001) and Ret is exclusively (Jain et al., 2006) expressed in DA neurons in the midbrain. We also show that the activation of the MAPK pathway is required for GDNF's action on the firing activity of DA neurons as well as for DA release. These results are in line with previous study in which we observed that infusion of GDNF into the VTA results in the rapid activation of ERK1/2 in VTA DA neurons (Carnicella et al., 2008). The fast action of GDNF on the neuronal firing of VTA DA neurons suggests a nongenomic, posttranslational mechanism. One possibility is the phosphorylation of an extranuclear substrate of ERK1/2. The spontaneous firing frequency of DA neurons is controlled mainly by subthreshold ionic currents (Bean, 2007), and several ion channels including the A-type $\mathrm{K}^{+}$channels (Liss et al., 2001) and voltagegated $\mathrm{Ca}^{2+}$ channels (Puopolo et al., 2007) are activated at subthreshold potentials. However, we did not observe ERK1/2mediated phosphorylation of Kv4.3, the A-type K channel that is expressed in the midbrain (data not shown). Another candidate is the voltage-gated $\mathrm{Ca}^{2+}$ channel. The activity of this channel is acutely enhanced by GDNF in cultured midbrain DA neurons (Wang et al., 2003) and in dorsal root ganglion neurons (Woodall et al., 2008), and several of the voltage-gated $\mathrm{Ca}^{2+}$ channel subunits contain putative MAPK phosphorylation sites (Martin et al., 2006). It is also plausible that ERK1/2 leads to the activation of a downstream kinase that will, in turn, phosphorylate one of these channels or others. Other posttranslational modifications such as lipid modifications and alterations in protein-protein interactions could also contribute to this rapid effect.

\section{Possible implications of NAc-derived GDNF regulating the spontaneous firing rate of NAc- and PFC-projecting VTA neurons}

The spontaneous firing of VTA DA neurons provides a DAergic tone in target areas of the mesocorticolimbic system, which was shown to be critical for cognitive functions such as working memory, planning, attention; for motivation to seek reward; and for general goal-directed behaviors (Nicola, 2007; Niv et al., 2007;
Schultz, 2007). Therefore, GDNF may play an important role in these cognitive functions, and this possibility merits further investigation.

In addition, DA in the NAc and PFC is also an important component of the neuronal circuitries underlying addiction (Hyman et al., 2006). By acting directly in the NAc or indirectly by enhancing the activity of VTA DA neurons, most drugs of abuse increase extracellular DA concentrations within the NAc, and repeated exposure to these drugs leads to substantial biochemical changes in the mesolimbic system (Hyman et al., 2006). Importantly, some of these neuroadaptations alter the activity of VTA DA neurons, resulting in a decrease in NAc DA levels during withdrawal from the drugs (Koob and Le Moal, 2001; Hyman et al., 2006). This DA hypofunction is suggested to lead to an increased motivation to seek and consume drugs of abuse to compensate for the deficit in DA and the associated negative emotional state (Koob and Le Moal, 2001). Interestingly, GDNF dampens adaptations resulting from exposure to drugs of abuse (Carnicella and Ron, 2009). Therefore, it is plausible that GDNF application in the VTA may reverse the DA hypofunction and, as a consequence, dramatically reduce drug-seeking and intake.

The mesolimbic DA pathway has also been implicated in depression (Nestler and Carlezon, 2006). Specifically, hypofunction of this system after chronic stress causes a reduction in responding for rewards in animal models of anhedonia and helplessness, two major symptoms of depression in humans (Cabib and Puglisi-Allegra, 1996). Interestingly, the mRNA (Otsuki et al., 2008) and protein (Zhang et al., 2008) levels of GDNF are reduced in patients suffering from major depression, suggesting that the spontaneous firing frequency of DA neurons in the VTA in depression is reduced. Conversely, in cell culture systems, several antidepressants such as amitriptyline increase the synthesis and secretion of GDNF (Hisaoka et al., 2001). Similarly, treatment with antidepressants was found to increase GDNF levels in the serum of patients suffering from depression (Zhang et al., 2008). Thus, the antidepressant-mediated increase in GDNF expression may restore the spontaneous firing activity of these neurons and ameliorate symptoms of depression.

In summary, our findings suggest that NAc-produced GDNF alters the spontaneous activity of VTA DA neurons that project to two distinct brain regions (the NAc and PFC). This long-distance retrograde GDNF-mediated enhancement in spontaneous firing activity, which leads to the increase of transmitter release, is likely to contribute to the regulation and maintenance of DA tone.

\section{References}

Airaksinen MS, Saarma M (2002) The GDNF family: signalling, biological functions and therapeutic value. Nat Rev Neurosci 3:383-394.

Barroso-Chinea P, Cruz-Muros I, Aymerich MS, Rodriguez-Diaz M, AfonsoOramas D, Lanciego JL, Gonzalez-Hernandez T (2005) Striatal expression of GDNF and differential vulnerability of midbrain dopaminergic cells. Eur J Neurosci 21:1815-1827.

Bean BP (2007) The action potential in mammalian central neurons. Nat Rev Neurosci 8:451-465.

Cabib S, Puglisi-Allegra S (1996) Stress, depression and the mesolimbic dopamine system. Psychopharmacology (Berl) 128:331-342.

Carnicella S, Ron D (2009) GDNF-a potential target to treat addiction. Pharmacol Ther 122:9-18.

Carnicella S, Kharazia V, Jeanblanc J, Janak PH, Ron D (2008) GDNF is a fast-acting potent inhibitor of alcohol consumption and relapse. Proc Natl Acad Sci U S A 105:8114-8119.

Carnicella S, Ahmadiantehrani S, He DY, Nielsen CK, Bartlett SE, Janak PH, Ron D (2009) Cabergoline decreases alcohol drinking and seeking behaviors via glial cell line-derived neurotrophic factor. Biol Psychiatry 66:146-153. 
Deierborg T, Soulet D, Roybon L, Hall V, Brundin P (2008) Emerging restorative treatments for Parkinson's disease. Prog Neurobiol 85:407-432.

Grace AA, Bunney BS (1984) The control of firing pattern in nigral dopamine neurons: single spike firing. J Neurosci 4:2866-2876.

Granholm AC, Reyland M, Albeck D, Sanders L, Gerhardt G, Hoernig G, Shen L, Westphal H, Hoffer B (2000) Glial cell line-derived neurotrophic factor is essential for postnatal survival of midbrain dopamine neurons. J Neurosci 20:3182-3190.

Hahn J, Tse TE, Levitan ES (2003) Long-term $\mathrm{K}^{+}$channel-mediated dampening of dopamine neuron excitability by the antipsychotic drug haloperidol. J Neurosci 23:10859-10866.

He DY, Ron D (2006) Autoregulation of glial cell line-derived neurotrophic factor expression: implications for the long-lasting actions of the antiaddiction drug, Ibogaine. FASEB J 20:2420-2422.

Henderson CE, Phillips HS, Pollock RA, Davies AM, Lemeulle C, Armanini M, Simmons L, Moffet B, Vandlen RA, Simpson LC, et al. (1994) GDNF: a potent survival factor for motoneurons present in peripheral nerve and muscle. Science 266:1062-1064.

Hisaoka K, Nishida A, Koda T, Miyata M, Zensho H, Morinobu S, Ohta M, Yamawaki S (2001) Antidepressant drug treatments induce glial cell line-derived neurotrophic factor (GDNF) synthesis and release in rat C6 glioblastoma cells. J Neurochem 79:25-34.

Hyman SE, Malenka RC, Nestler EJ (2006) Neural mechanisms of addiction: the role of reward-related learning and memory. Annu Rev Neurosci 29:565-598.

Jain S, Golden JP, Wozniak D, Pehek E, Johnson EM Jr, Milbrandt J (2006) RET is dispensable for maintenance of midbrain dopaminergic neurons in adult mice. J Neurosci 26:11230-11238.

Johnson SW, Seutin V, North RA (1992) Burst firing in dopamine neurons induced by $\mathrm{N}$-methyl-D-aspartate: role of electrogenic sodium pump. Science 258:665-667.

Joyce EM, Stinus L, Iversen SD (1983) Effect of injections of 6-OHDA into either nucleus accumbens septi or frontal cortex on spontaneous and drug-induced activity. Neuropharmacology 22:1141-1145.

Keefe KA, Zigmond MJ, Abercrombie ED (1993) In vivo regulation of extracellular dopamine in the neostriatum: influence of impulse activity and local excitatory amino acids. J Neural Transm Gen Sect 91:223-240.

Khaliq ZM, Bean BP (2010) Pacemaking in dopaminergic ventral tegmental area neurons: depolarizing drive from background and voltagedependent sodium conductances. J Neurosci 30:7401-7413.

Kobori N, Waymire JC, Haycock JW, Clifton GL, Dash PK (2004) Enhancement of tyrosine hydroxylase phosphorylation and activity by glial cell line-derived neurotrophic factor. J Biol Chem 279:2182-2191.

Koob GF, Le Moal M (2001) Drug addiction, dysregulation of reward, and allostasis. Neuropsychopharmacology 24:97-129.

Lammel S, Hetzel A, Hackel O, Jones I, Liss B, Roeper J (2008) Unique properties of mesoprefrontal neurons within a dual mesocorticolimbic dopamine system. Neuron 57:760-773.

Lauterborn JC, Rex CS, Kramar E, Chen LY, Pandyarajan V, Lynch G, Gall CM (2007) Brain-derived neurotrophic factor rescues synaptic plasticity in a mouse model of fragile X syndrome. J Neurosci 27:10685-10694.

Ledda F, Paratcha G, Sandoval-Guzman T, Ibanez CF (2007) GDNF and GFR $\alpha 1$ promote formation of neuronal synapses by ligand-induced cell adhesion. Nat Neurosci 10:293-300.

Lin LF, Doherty DH, Lile JD, Bektesh S, Collins F (1993) GDNF: a glial cell line-derived neurotrophic factor for midbrain dopaminergic neurons. Science 260:1130-1132.

Liss B, Franz O, Sewing S, Bruns R, Neuhoff H, Roeper J (2001) Tuning pacemaker frequency of individual dopaminergic neurons by $\mathrm{Kv} 4.3 \mathrm{~L}$ and KChip3.1 transcription. EMBO J 20:5715-5724.

Lu L, Wang X, Wu P, Xu C, Zhao M, Morales M, Harvey BK, Hoffer BJ, Shaham Y (2009) Role of ventral tegmental area glial cell line-derived neurotrophic factor in incubation of cocaine craving. Biol Psychiatry 66:137-145.

Lu XY, Ghasemzadeh MB, Kalivas PW (1998) Expression of D1 receptor, $D 2$ receptor, substance $P$ and enkephalin messenger RNAs in the neurons projecting from the nucleus accumbens. Neuroscience 82:767-780.

Magby JP, Bi C, Chen ZY, Lee FS, Plummer MR (2006) Single-cell characterization of retrograde signaling by brain-derived neurotrophic factor. J Neurosci 26:13531-13536.

Margolis EB, Mitchell JM, Ishikawa J, Hjelmstad GO, Fields HL (2008) Mid- brain dopamine neurons: projection target determines action potential duration and dopamine $\mathrm{D}_{2}$ receptor inhibition. J Neurosci 28:89088913.

Martin SW, Butcher AJ, Berrow NS, Richards MW, Paddon RE, Turner DJ, Dolphin AC, Sihra TS, Fitzgerald EM (2006) Phosphorylation sites on calcium channel $\alpha 1$ and $\beta$ subunits regulate ERK-dependent modulation of neuronal N-type calcium channels. Cell Calcium 39:275-292.

Nestler EJ, Carlezon WA Jr (2006) The mesolimbic dopamine reward circuit in depression. Biol Psychiatry 59:1151-1159.

Nicola SM (2007) The nucleus accumbens as part of a basal ganglia action selection circuit. Psychopharmacology (Berl) 191:521-550.

Niv Y, Daw ND, Joel D, Dayan P (2007) Tonic dopamine: opportunity costs and the control of response vigor. Psychopharmacology (Berl) 191: 507-520.

Oo TF, Ries V, Cho J, Kholodilov N, Burke RE (2005) Anatomical basis of glial cell line-derived neurotrophic factor expression in the striatum and related basal ganglia during postnatal development of the rat. J Comp Neurol 484:57-67.

Otsuki K, Uchida S, Watanuki T, Wakabayashi Y, Fujimoto M, Matsubara T, Funato H, Watanabe Y (2008) Altered expression of neurotrophic factors in patients with major depression. J Psychiatr Res 42:1145-1153.

Pascual A, Hidalgo-Figueroa M, Piruat JI, Pintado CO, Gomez-Diaz R, Lopez-Barneo J (2008) Absolute requirement of GDNF for adult catecholaminergic neuron survival. Nat Neurosci 11:755-761.

Pochon NA, Menoud A, Tseng JL, Zurn AD, Aebischer P (1997) Neuronal GDNF expression in the adult rat nervous system identified by in situ hybridization. Eur J Neurosci 9:463-471.

Puopolo M, Raviola E, Bean BP (2007) Roles of subthreshold calcium current and sodium current in spontaneous firing of mouse midbrain dopamine neurons. J Neurosci 27:645-656.

Regehr WG, Carey MR, Best AR (2009) Activity-dependent regulation of synapses by retrograde messengers. Neuron 63:154-170.

Sarabi A, Hoffer BJ, Olson L, Morales M (2001) GFR $\alpha-1$ mRNA in dopaminergic and nondopaminergic neurons in the substantia nigra and ventral tegmental area. J Comp Neurol 441:106-117.

Schultz W (2007) Multiple dopamine functions at different time courses. Annu Rev Neurosci 30:259-288.

Swanson LW (1982) The projections of the ventral tegmental area and adjacent regions: a combined fluorescent retrograde tracer and immunofluorescence study in the rat. Brain Res Bull 9:321-353.

Tomac A, Lindqvist E, Lin LF, Ogren SO, Young D, Hoffer BJ, Olson L (1995a) Protection and repair of the nigrostriatal dopaminergic system by GDNF in vivo. Nature 373:335-339.

Tomac A, Widenfalk J, Lin LF, Kohno T, Ebendal T, Hoffer BJ, Olson L (1995b) Retrograde axonal transport of glial cell line-derived neurotrophic factor in the adult nigrostriatal system suggests a trophic role in the adult. Proc Natl Acad Sci U S A 92:8274-8278.

Trupp M, Belluardo N, Funakoshi H, Ibanez CF (1997) Complementary and overlapping expression of glial cell line-derived neurotrophic factor (GDNF), c-ret proto-oncogene, and GDNF receptor- $\alpha$ indicates multiple mechanisms of trophic actions in the adult rat CNS. J Neurosci 17: 3554-3567.

Wang J, Chen G, Lu B, Wu CP (2003) GDNF acutely potentiates $\mathrm{Ca}^{2+}$ channels and excitatory synaptic transmission in midbrain dopaminergic neurons. Neurosignals 12:78-88.

Woodall AJ, Richards MA, Turner DJ, Fitzgerald EM (2008) Growth factors differentially regulate neuronal Cav channels via ERK-dependent signalling. Cell Calcium 43:562-575.

Yang F, Feng L, Zheng F, Johnson SW, Du J, Shen L, Wu CP, Lu B (2001) GDNF acutely modulates excitability and A-type $\mathrm{K}^{+}$channels in midbrain dopaminergic neurons. Nat Neurosci 4:1071-1078.

Zapata A, Shippenberg TS (2005) Lack of functional D2 receptors prevents the effects of the D3-preferring agonist (+)-PD 128907 on dialysate dopamine levels. Neuropharmacology 48:43-50.

Zhang X, Poo MM (2002) Localized synaptic potentiation by BDNF requires local protein synthesis in the developing axon. Neuron 36: $675-688$.

Zhang X, Zhang Z, Xie C, Xi G, Zhou H, Zhang Y, Sha W (2008) Effect of treatment on serum glial cell line-derived neurotrophic factor in depressed patients. Prog Neuropsychopharmacol Biol Psychiatry 32:886890. 